\title{
Combined Use of Genetic Algorithms and Gradient Descent Optmization Methods for Accurate Inverse Permittivity Measurement
}

\author{
Maria E. Requena-Pérez, Antonio Albero-Ortiz, Juan Monzó-Cabrera, and Alejandro Díaz-Morcillo, Member, IEEE
}

\begin{abstract}
A novel inverse transmission-line method for the complex permittivity determination of arbitrary shaped materials is presented. Complex permittivity is inferred by using an inverse calculation procedure, which is based on a combined optimization strategy of both genetic algorithms and the gradient descent method. The optimization procedure matches the measured and simulated complex scattering parameters' frequency behavior of materials within a WR340 waveguide. High accuracy and practical suitability are validated through experimental tests. The dielectric properties of PTFE and epoxy resin mixed with iron-oxide-doped fiberglass have been measured for different shapes and positions. Dielectric multilayer structures have been used to demonstrate that this technique is able to measure the individual permittivity of each element of the structure. Both twoand three-dimensional approaches have been carried out and their advantages and drawbacks discussed.
\end{abstract}

Index Terms-Complex permittivity measurement, genetic algorithms (GAs), gradient descent (GD) method, microwave energy.

\section{INTRODUCTION}

$\mathbf{T}$ HE knowledge of the complex permittivity is a key point in a great number of applications. In fact, the design of devices or systems in several microwave applications such as communications, microelectronics, radar, or industrial microwave heating [1], [2] requires the precise permittivity determination of the involved dielectric materials.

During the last decades, many methods have been developed, analyzed, and employed for measuring the complex permittivity of dielectrics [3]. The most used measuring techniques are based on cavity perturbation, transmission lines, and free-space configurations [4]. Cavity or perturbational techniques have been used for low-loss materials successfully. Although these techniques provide good accuracy values in this case, the size and permittivity of the sample limit them, since the perturbation must be minimum and, moreover, the results are obtained only at discrete frequencies. In transmission-line techniques, on the contrary, the dielectric and magnetic properties can be determined over a wide frequency range by measuring the scattering parameters of a coaxial or waveguide structure with a dielectric sample completely [5]-[8] or partially [9] filling the cross

Manuscript received April 11, 2005; revised July 5, 2005. This work was supported in part by the Comisión Interministerial de Ciencia y Tecnología, Spain, under Project TIC2004-05037-C02-02.

The authors are with the Departamento de Tecnologías de la Información y las Comunicaciones, Technical University of Cartagena, 30203 Cartagena (Murcia), Spain (e-mail: mae.requena@upct.es).

Digital Object Identifier 10.1109/TMTT.2005.862671 section. In contrast, the precision of transmission/reflection methods for low-loss materials is restricted by the uncertainty of the network analyzer. Furthermore, the waveguide or coaxial-line end methods require a homogeneous and very tightly contacting sample. The same algorithms that are used by transmission-line methods can be used by the free-space methods [10]. These methods use large samples, and, normally, they are nondestructive technique but suffer from reduced accuracy because of unwanted reflections of the surrounding object and the diffraction from the edges of the sample.

Generally, direct methods are quick, but sometimes they do not provide the required accuracy. Moreover, these methods need the sample to have a canonical shape and, when a multilayer material must be measured in order to obtain the individual permittivities of its elements, they obtain an effective permittivity but are not able to measure the individual permittivity of each layer.

Several inverse techniques have been previously employed to determine both the complex permittivity and/or permeability values. In [11], the calculation of dielectric and magnetic properties is made by an electromagnetic analysis of the test microstrip device together with a numerical optimization based on gradient method. In [12], a nonreciprocal waveguide cell is employed to measure the permeability through the use of the Levenberg-Marquardt method. Monte Carlo simulation and the finite-element method (FEM) are used in order to extract the permittivity of arbitrary materials within a microwave cavity in [13]. Complex permittivity is also reconstructed in [14] by using neural networking inverse measurement procedures. Additionally, the Newton-Raphson method has been employed in order to match experimental data and computed values of frequency shift and quality factor changes in cavity permittivity measurements [15]. This optimization technique has also been used for the permittivity inverse estimation of dielectrics within short-circuited waveguides [16].

Genetic algorithms (GAs) have been used in the last few years by the electromagnetic community as an optimization technique [17]. GAs are proving to be useful for solving complex electromagnetic problems such as electromagnetic devices design [18]-[20]. Regarding the specific use of GAs in permittivity inverse measurements, a free-space multilayer structure evaluation has been carried by using analytical equations in [21]. The GA and the method of moments (MoM) have been simultaneously applied in [22] and [23] to measure the dielectric constant of rectangular strips within rectangular waveguides by comparing the measured and computed reflection coefficients. 
Furthermore, several studies have shown the successful use of coaxial sensors jointly with GA techniques to predict the permittivity of both saponification reactions [24] and solid materials [25].

Recently, the combination of GA and three-dimensional (3-D) electromagnetic (EM) commercial software based on the finite-integration technique (FIT) has proven that high-precision waveguide measurements can be carried out [26], although this approach suffers from high computing times due to both the high amount of generations needed by the GA to achieve convergence and the huge amount of computing resources employed by 3-D electromagnetic simulators.

In this study, an iterative inverse method for the complex permittivity measurement of arbitrarily shaped samples within a waveguide is presented. In fact, some complex sample geometries used in this study, such as E-shaped or cylinder samples, have not been measured in transmission/reflection waveguide measurements yet since previous studies usually handle simpler sample geometries [22], [23], [26]. Additionally, dielectric multilayer measurements have been carried out to show how this technique can be employed to infer the permittivity of each element in the dielectric structure. Therefore, the waveguide measurement setups, evaluation functions, optimization strategies, and numerical methods employed in previous studies [22], [23] differ greatly from the ones proposed in this study.

The inverse technique uses an in-house-made EM simulator based on FEM for two-dimensional (2-D) structures. For 3-D scenarios, a FIT commercial software is used [28]. Both 2-D and 3-D EM simulators are used for computing the EM fields and the scattering parameters of waveguide structures that contain a dielectric sample with an arbitrary geometry. These computed scattering parameters are then compared to the measured ones through the definition of an objective error function that must be minimized. In this case, a hybrid technique is used for minimizing the objective function and to reduce computing times. As a difference with other studies [26], this hybrid technique is based on the combined strategy of the GA and the gradient-descent (GD) method in order to combine the advantages of both optimization techniques. Furthermore, the results obtained by 2-D and 3-D software are compared in terms of accuracy and computational cost.

\section{INVERSE PROBLEM}

In this study, we have observed several WR-340 waveguide measurement cells containing different dielectric samples with arbitrary shapes and positions. In order to compare both 2-D and 3-D approaches, we have restricted the sample shapes to provide invariance along the shorter dimension of the waveguide cross section. However, the proposed algorithm could work with any kind of sample size, shape, or position by using the proposed 3-D approach.

The hybrid inverse technique reconstructs the permittivity frequency behavior of arbitrarily shaped dielectric samples by minimizing the difference between the measured and the computed scattering parameters. Thus, this technique includes three interrelated stages: the modeling of the waveguide EM behavior as a function of the sample permittivity (direct problem), the scattering matrix measurement of the waveguide cell filled with the dielectric, and the matching of the measured and computed scattering parameters (inverse problem) by using an optimization procedure.

The relationship between the scattering parameters and complex permittivity is not straightforward since the shape and position of the sample is only restricted to keep constant along one space direction. As a consequence, the calculation of the dielectric properties from the measured scattering parameters requires an optimization method that uses the EM simulation of the problem under test.

\section{A. Scattering Parameter Computation: 2-D Approach}

An in-house 2-D FEM-based software has been employed to solve the wave equation

$$
-\nabla \cdot\left(\frac{1}{\mu} \nabla \vec{E}\right)-\omega^{2} \varepsilon \vec{E}=0
$$

for each electric field component, by using the variational formulation as indicated in [27] where $\vec{E}$ is the vector electric field, $\omega$ is the angular frequency, $\mu$ is the magnetic permeability, and $\varepsilon$ is the dielectric complex permittivity of the medium defined as

$$
\varepsilon=\varepsilon_{o}\left(\varepsilon^{\prime}-j \varepsilon^{\prime \prime}\right)=\varepsilon_{o} \varepsilon^{\prime}(1-j \tan \delta)
$$

where $\varepsilon_{o}$ is the vacuum permittivity, $\varepsilon^{\prime}$ is the dielectric constant, $\varepsilon^{\prime \prime}$ is the loss factor, and $\tan \delta$ the loss tangent.

This 2-D software is based on the online programming of the PDE MATLAB toolbox [27], allowing the $\mathrm{TE}_{10}$ mode excitation at the corresponding port and the definition of an absorbing boundary condition to provide adaptation in the other port through the use of a general Neumann boundary condition. In this way, the scattering parameters of the waveguide cell can be computed from the electric field computation as

$$
\begin{aligned}
& s_{11}=\frac{E(0)-E_{i}}{E_{i}} \mid \text { port } 2 \text { adapted } s_{21}=\left.\frac{E(L)}{E_{i}}\right|_{\text {port } 2 \text { adapted }} \\
& s_{22}=\frac{E(L)-E_{j}}{E_{j}} \mid \text { port } 1 \text { adapted } s_{12}=\left.\frac{E(0)}{E_{j}}\right|_{\text {port } 1 \text { adapted }}
\end{aligned}
$$

where $E(0)$ is the total electric field at the center of port 1, $E(L)$ is the total electric field at the center of port 2, and $E_{i}$ and $E_{j}$ are the incident electric fields at ports 1 and 2, respectively.

It is important to remark that the 2-D approach can be used to compute the scattering parameters because the $\mathrm{TE}_{10}$ mode is invariable across the smaller dimension of the WR-340 waveguide and due to the fact that the dielectric sample geometry keeps constant along this dimension.

\section{B. Scattering Parameter Computation: 3-D Approach}

A more general approach is the use of 3-D EM simulators to compute the scattering parameters. These programs permit any kind of sample positions or shapes within the waveguide holder. 
In this case, the CST Microwave Studio 4.0 (CST MWS) commercial software has been used in order to compute the scattering parameters of the 3-D schemes of both the holder and the dielectric sample.

CST MWS is a general-purpose electromagnetic simulator based on the FIT first proposed by Weiland [28]. FIT provides a general spatial discretization scheme that is applicable to various electromagnetic problems, such as static field calculations or high-frequency applications in the time or frequency domains.

\section{Description of Optimization Techniques}

The proposed optimization procedures used to carry out the permittivity measurements are based on GA, GD, and the combined strategy of both methods in order to make best use of their specific advantages.

GA optimization methods are robust, stochastic search techniques modeled on the principles and concepts of natural selection and evolution. GAs are particularly effective when the goal is to find a global optimum, which, in this case, is the complex permittivity. In GAs, a population of potential solutions is caused to evolve toward a global optimal solution. The evolution toward a global optimum occurs as a result of two processes: on the one hand, the pressure exerted by a fitness-weighted selection process, and, on the other hand, the exploration of the solution space, which is accomplished by recombination and mutation of existing characteristics present in the current population [17]. The best individual of the last generation is the start point for the GD. GA optimization methods have been implemented as MATLAB function [29].

Gradient methods are generally efficient when the function to be optimized is continuous in its first derivative. These methods use information about the slope of the function to follow a search direction of the minimum. The best advantage of GD methods is that convergence can be very fast if the error function shows high gradients toward the global minimum. However, GD may get stuck on local minima. In this study, a multivariable optimization MATLAB function that implements the quasi-Newton method has been employed [30].

In this study, we suggest a hybrid optimization technique $(\mathrm{GA}+\mathrm{GD})$ that employs the GA and GD at different stages of the search. The GA initiates the search and locates the zone of the global optimum, and then the GD searches the optimum [31]. Starting from this improved initial point, this search quickly converges. Obviously, it is assumed at this stage that, close to the global optimum, the objective function is differentiable and without local minima. This hybrid optimization technique has also been compared in terms of accuracy and computing times to both pure GA and GD optimization approaches.

Fig. 1 shows the flowchart of the hybrid technique. First, an initial population is randomly generated. A population is a group of potential solutions or individuals, $\left\{x_{1}, \ldots, x_{k}\right\}$. Each individual consists of a sequence of genes representing the unknown parameters. In this case, the genes are the dielectric constant and the loss tangent of the sample, $x_{i}=\left[\varepsilon^{\prime}, \tan \delta\right]$, and the generation is the iteration number during GA optimization.

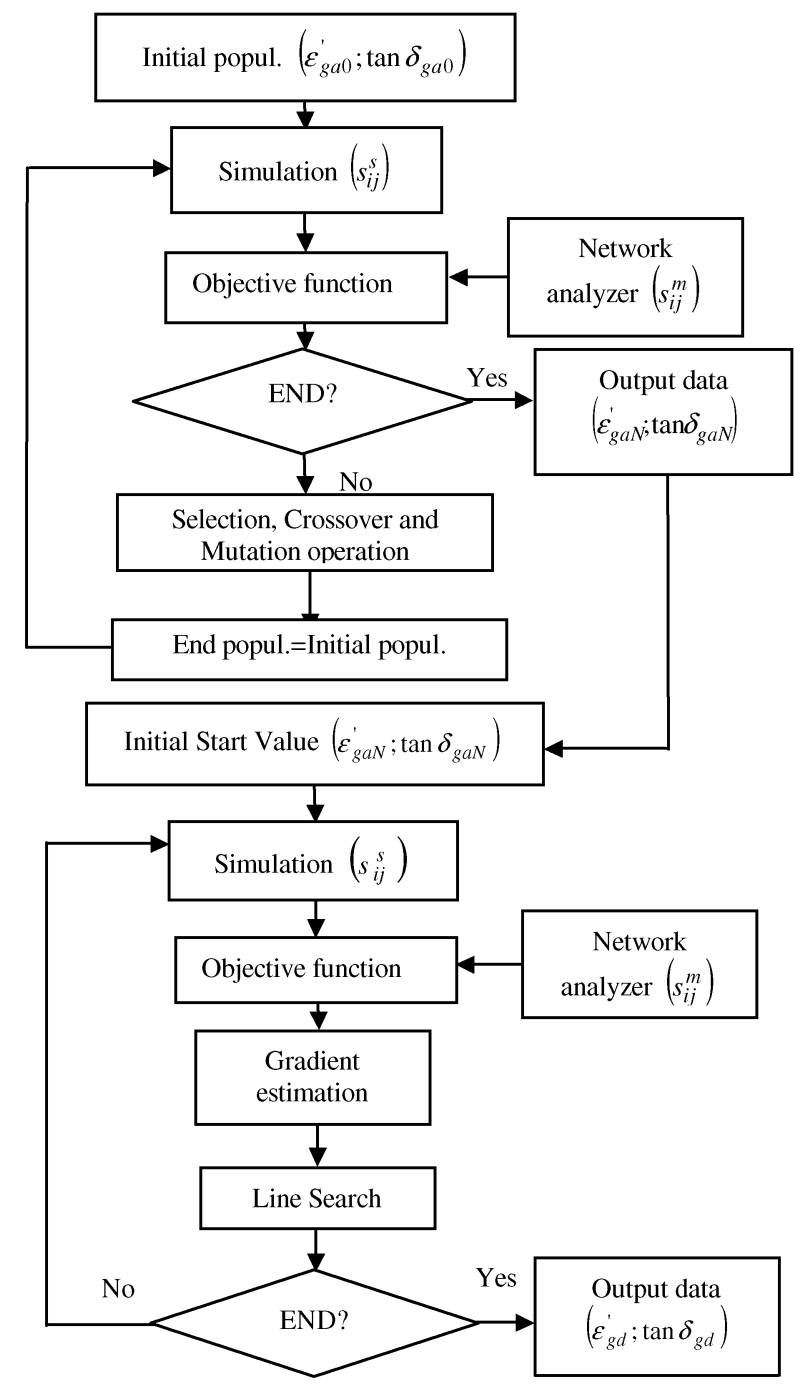

Fig. 1. Flowchart of the hybrid technique.

The objective function evaluates the difference between simulated and measured scattering parameters, and this fitness is assigned to the individual. Then, the Selection, Crossover, and Mutation operators are applied to results of each population until the solution converges [17]. The termination condition is the number of generations $g_{\max }=N$.

The objective function used in this study is [24]

$$
\begin{aligned}
F= & p \cdot\left(\sum_{i=1}^{2} \sum_{j=1}^{2} \sqrt{\left.\sum_{k=1}^{N}\left(\left|s_{i j}^{m}(k)\right|-\left|s_{i j}^{s}(k)\right|\right)^{2}\right)}\right. \\
& \left.+(1-p)\left(\sum_{i=1}^{2} \sum_{j=1}^{2} \sqrt{\sum_{k=1}^{N}\left(\theta\left(s_{i j}^{m}(k)\right)-\theta\left(s_{i j}^{s}(k)\right)\right)^{2}}\right)\right)
\end{aligned}
$$

where $p \in[0,1]$ is a weighting factor, $s_{i j}^{s}$, with $i=1,2$ and $j=1,2$, are simulated scattering parameters expressed as linear magnitudes, $N$ is the number of evaluated frequencies, $s_{i j}^{m}$, with $i=1,2$ and $j=1,2$, are measured scattering parameters, and $\theta\left(s_{i j}^{m}(k)\right)$ and $\theta\left(s_{i j}^{s}(k)\right)$ are the measured or computed phase 


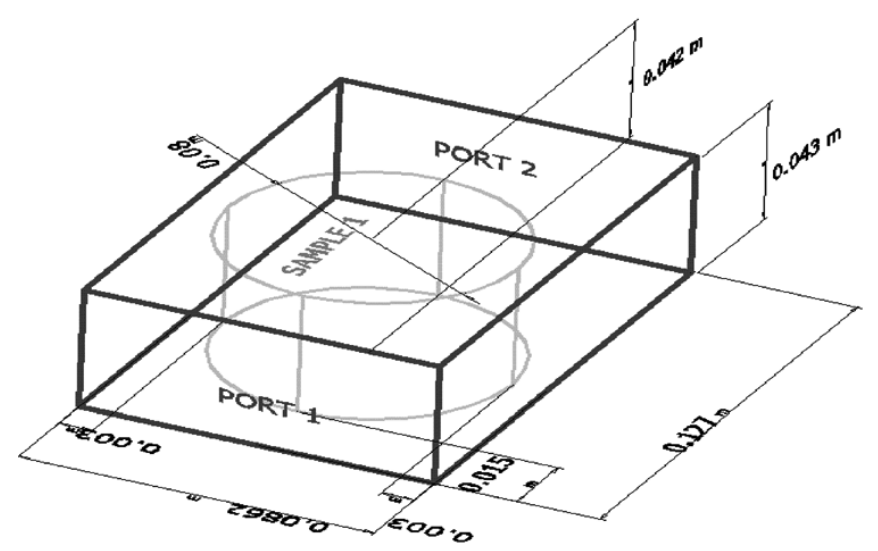

Fig. 2. Sample 1 geometry and position in waveguide holder. M1.

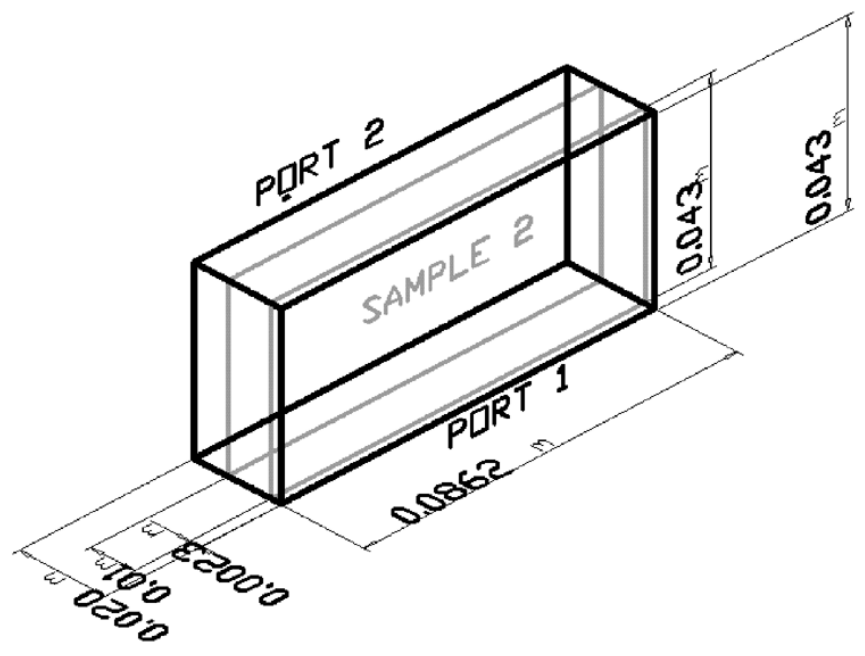

Fig. 3. Sample 2 geometry and position in waveguide holder. M1.

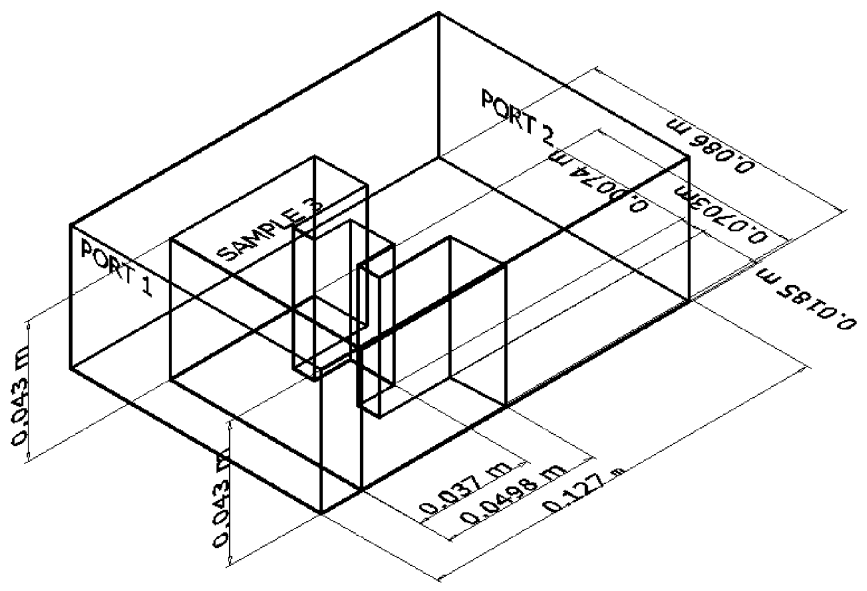

Fig. 4. Sample 3 geometry and position in waveguide holder. M1.

of scattering parameters, respectively. In this study, $p$ has been fixed to 0.5 for all simulations.

\section{EXPERIMENTAL SETUP}

An Agilent 8720 vector network analyzer and several WR340 rectangular waveguides have been used to measure the scattering parameters $s_{i j}^{m}$. Figs. 2-5 show the different samples'

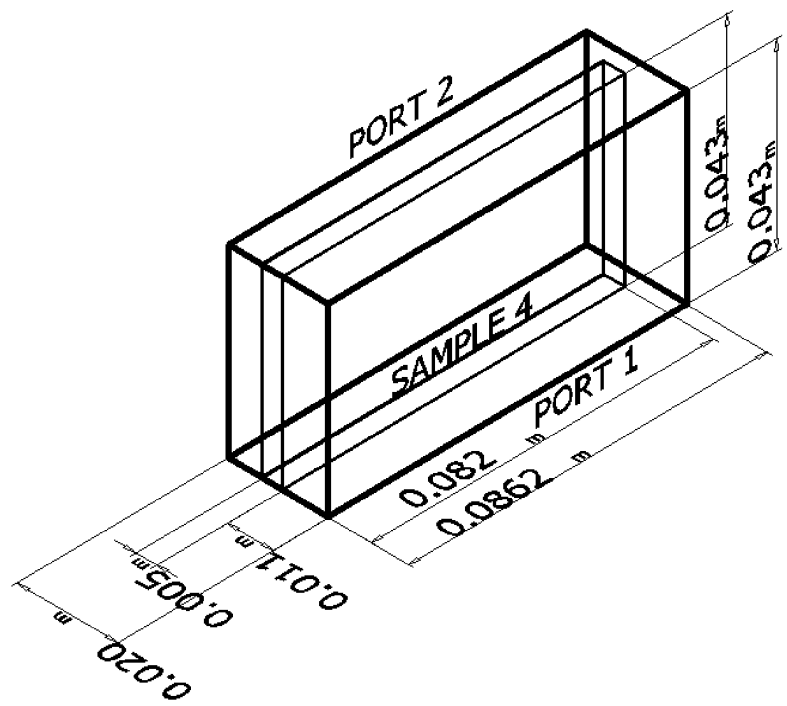

Fig. 5. Sample 4 geometry and position in waveguide holder. M2.

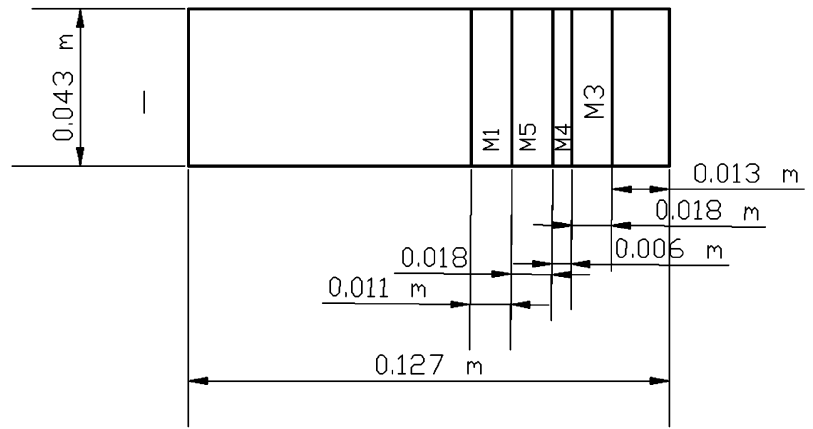

Fig. 6. Dielectric multilayer structure and position in waveguide holder. ML1.

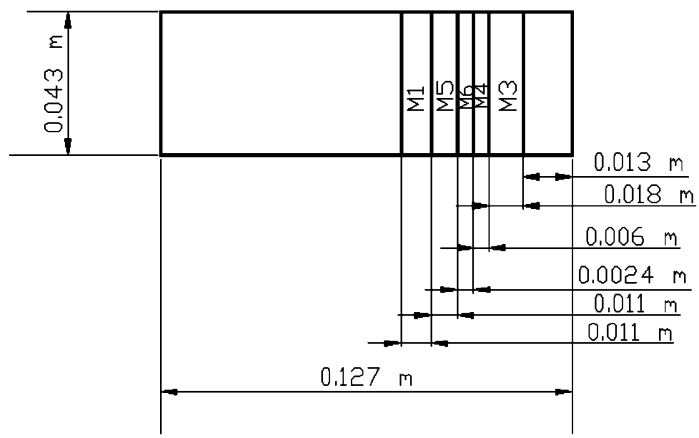

Fig. 7. Dielectric multilayer structure and position in waveguide holder. ML2.

geometry and waveguide holders used during measurements. Sample 1 was a PTFE cylinder, while samples 2 and 4 were mechanized as two rectangular slabs of PTFE and epoxy resin mixed with fiberglass doped with iron-oxide materials, respectively. In sample 3, the PTFE was shaped into a capital E. Figs. 6 and 7 show the dielectric multilayer structures within the waveguide. In Fig. 6, the multilayer 1 (ML1) is composed of PTFE, fir wood, styrofoam, and polyester resin. Multilayer 2 (ML2) in Fig. 7 has five components: PTFE, Styrofoam, polycarbonate, fir wood, and epoxy resin. In both structures, all of the materials that make up the structure completely fill the waveguide cross section. 
TABLE I

Permitivity Results For SAMPle 1 (M1, Cylinder)

\begin{tabular}{|c|c|c|c|c|c|c|c|c|}
\hline \multirow[t]{2}{*}{ Method } & \multirow{2}{*}{$\begin{array}{c}\text { Termination Condition } \\
-- \\
\end{array}$} & \multicolumn{2}{|c|}{ Start Point } & \multicolumn{2}{|c|}{ Measurement } & \multirow[t]{2}{*}{ Error } & \multirow[t]{2}{*}{ Time } & \multirow{2}{*}{$\begin{array}{c}\text { Iterations/ } \\
\text { Generation } \\
s \\
\end{array}$} \\
\hline & & $\varepsilon^{\prime}$ & $\varepsilon^{\prime \prime}$ & $\varepsilon^{\prime}$ & $\varepsilon^{\prime \prime}$ & & & \\
\hline 87051 & -- & & & 2.1 & 0.01 & & & \\
\hline Literature [2] & -- & & & 2.1 & 0.0003 & & & \\
\hline \multirow[b]{2}{*}{ GD1 } & Iteration $\max =10$ & 6.56 & 3.92 & 8.17 & 0.02 & 4.5067 & 3'50', & 10 \\
\hline & $\begin{array}{l}\mathcal{E}^{\prime} \in[2.096 \pm 0.042] \& \\
\varepsilon^{\prime \prime} \in[0.0118 \pm 0.0002]\end{array}$ & 6.56 & 3.92 & -- & -- & -- & $\infty$ & $\infty$ \\
\hline \multirow[b]{2}{*}{ GD2 } & Iteration $\max =10$ & 2.51 & 0.7 & 2.04 & 0.04 & 1.7412 & $10^{\prime} 33^{\prime \prime}$ & 10 \\
\hline & $\begin{array}{c}\varepsilon^{\prime} \in[2.096 \pm 0.042] \& \\
\mathcal{E}^{\prime \prime} \in[0.0118 \pm 0.0002]\end{array}$ & 2.51 & 0.7 & 2.056 & 0.012 & 0.7416 & $37^{\prime} 53^{\prime \prime}$ & 41 \\
\hline \multirow[b]{2}{*}{ GA } & Gmax $=25$ & -- & -- & 2.108 & 0.036 & 0.7253 & $77^{\prime} 47^{\prime \prime}$ & 25 \\
\hline & $\begin{array}{l}\mathcal{E}^{\prime} \in[2.096 \pm 0.042] \& \\
\mathcal{E}^{\prime \prime} \in[0.0118 \pm 0.0002]\end{array}$ & -- & -- & 2.109 & 0.0122 & 0.6979 & $171^{\prime} 52^{\prime \prime}$ & 214 \\
\hline $\mathrm{GA}+\mathrm{GD}$ & Gmax $=25$ and iter $=10$ & -- & -- & 2.096 & 0.0118 & 0.6826 & $13^{\prime} 10^{\prime \prime}$ & $25+10$ \\
\hline
\end{tabular}

Consequently, six materials have been employed for dielectric properties estimation: PTFE (M1), epoxy resin mixed with fiberglass doped with iron oxide (M2), fir wood (M3), Styrofoam (M4), polyester resin (M5), and polycarbonate (M6). PTFE has been used for validation purposes due to its wide use in literature. The frequency bandwidth ranged from 2.2 to $3.3 \mathrm{GHz}$ for single materials' measurements and from 2 to $2.75 \mathrm{GHz}$ in the case of multilayer structures.

The use of these low-loss materials is due to the fact that waveguide measurements are not very precise when dielectric losses are low. Thus, we have chosen the worst case to test the proposed inverse measurement technique.

Finally, a $10 \times 2 \times 34 \mathrm{~cm}^{3}$ rectangular monomode cavity has been used in order to obtain additional data for the permittivity of the epoxy resin. The excited mode for the cavity was TE107 and the operating frequency set to around $3 \mathrm{GHz}$. In this case, the sample was a $0.234 \times 2 \times 1 \mathrm{~cm}^{3}$ rectangular sheet placed at the center of the cavity. For multilayer structure permittivity validation, the Nicholson-Ross method has been used [5] to measure the individual permittivity of each component.

\section{NumERICAL RESUlTS AND DISCUSSION}

Several inverse measurements have been performed to illustrate the accuracy and computational cost of the proposed technique in the evaluation of complex permittivity. In order to test the accuracy of the 2-D inverse procedure, the value of complex permittivity of both M1 and M2 dielectrics has been measured by using an Agilent 8720 Network Analyzer and the Agilent 87051 software, ${ }^{1}$ yielding $\varepsilon_{r}=2.1-\mathrm{j} 0.01$ for M1 and $\varepsilon_{r}=3.8327-\mathrm{j} 0.0385$ for M2. This commercial software uses the Nicholson-Ross technique to obtain the complex permittivity [5]. Since the resolution of waveguide methods for low-loss material is restricted by the uncertainty of the network analyzer, the permittivity of $\mathrm{M} 1$ is compared to the permittivity

\footnotetext{
${ }^{1}$ Agilent Technol., Innovative Measurement Solutions Inc., Help 85071, ver. D1.00, 1988-2001
}

TABLE II

PERMitivity Results FOR SAMPLE 2 (M1, ReCTANGULAR)

\begin{tabular}{lcccccc}
\hline \hline \multirow{2}{*}{ Method } & \multicolumn{2}{c}{ Start Point } & \multicolumn{2}{c}{ Measurement } & \multirow{2}{*}{ Error } & \multirow{2}{*}{ Time } \\
& $\varepsilon^{\prime}$ & $\varepsilon^{\prime \prime}$ & $\varepsilon^{\prime}$ & $\varepsilon^{\prime \prime}$ & & \\
\hline 87051 & & & 2.1 & 0.01 & & \\
\hline Literature & & & 2.1 & 0.0003 & & \\
[2] & & & & & \\
GD1 & 6.56 & 3.92 & $\mathbf{6 . 6 3}$ & $\mathbf{1 . 8 8}$ & 14.45 & $9^{\prime} 24^{\prime}$, \\
GD2 & 2.51 & 0.7 & $\mathbf{2 . 1 2}$ & $\mathbf{0 . 0 1 4}$ & 0.13 & $18^{\prime} 52^{\prime}$, \\
GA & -- & -- & $\mathbf{2 . 1 1}$ & $\mathbf{0 . 0 0 6 6}$ & 0.36 & $22^{\prime} 47^{\prime}$, \\
GA+GD & -- & -- & $\mathbf{2 . 0 9 6}$ & $\mathbf{0 . 0 1 2}$ & 0.13 & $20^{\prime} 24^{\prime}$, \\
\hline
\end{tabular}

TABLE III

PERMITIVITY RESUlTS FOR SAMPLE 3 (M1, E-SHAPED)

\begin{tabular}{|c|c|c|c|c|c|c|}
\hline \multirow{2}{*}{ Method } & \multicolumn{2}{|c|}{ Start Point } & \multicolumn{2}{|c|}{ Measurement } & \multirow{2}{*}{ Error } & \multirow{2}{*}{ Time } \\
\hline & $\varepsilon^{\prime}$ & $\varepsilon "$ & $\varepsilon^{\prime}$ & $\varepsilon^{\prime \prime}$ & & \\
\hline 87051 & & & 2.1 & 0.01 & & \\
\hline $\begin{array}{l}\text { Literature } \\
\text { [2] }\end{array}$ & & & 2.1 & 0.0003 & & \\
\hline GD1 & 6.56 & 3.92 & 8.28 & 2.72 & 25.49 & $41^{\prime} 57^{\prime}$, \\
\hline GD2 & 2.51 & 0.7 & 1.80 & 0.32 & 16.23 & $103^{\prime} 52^{\prime}$ ' \\
\hline GA & - & -- & 2.096 & 0.027 & 13.74 & $61^{\prime} 47^{\prime}$, \\
\hline $\mathrm{GA}+\mathrm{GD}$ & -- & -- & 2.064 & 0.033 & 7.78 & 153'20', \\
\hline
\end{tabular}

from literature [2], and the permittivity of $\mathrm{M} 2$ has been calculated through the use of a cavity measurement. All of the simulations were carried out in a personal computer with a $2.4-\mathrm{GHz}$ Pentium IV processor and 512-MB RAM memory.

\section{A. 2-D Inverse Measurements}

Tables I-IV show the complex permittivity, the error obtained by (5), and the computation time with different techniques, namely GD, GA and the proposed hybrid technique (GA+GD) for Samples 1-4, respectively. In all of these inverse measurements, the MATLAB 2-D in-house-made code was employed to model the waveguide cell behavior. In the particular case of Table I, two termination conditions are used: one is based on a maximum iteration number or maximum number of generations for GD or GA, respectively, and the other one is based on a required accuracy (2\%) around the optimum value provided by the GA + GD algorithm. 
TABLE IV

PERMITIVITY RESUlTS FOR SAMPLE 4 (M2, RECTANGULAR)

\begin{tabular}{|c|c|c|c|c|c|c|}
\hline \multirow{2}{*}{ Method } & \multicolumn{2}{|c|}{ Start Point } & \multicolumn{2}{|c|}{ Measurement } & \multirow{2}{*}{$\begin{array}{c}\text { Erro } \\
r\end{array}$} & \multirow{2}{*}{ Time } \\
\hline & $\varepsilon^{\prime}$ & $\varepsilon$, & $\varepsilon^{\prime}$ & $\varepsilon$, & & \\
\hline 87051 & & & 3.83 & 0.0385 & & \\
\hline $\begin{array}{l}\text { Cavity } \\
(3 \mathrm{GHz})\end{array}$ & & & 3.73 & 0.035 & & \\
\hline GD1 & 6.56 & 3.92 & 3.81 & 0.049 & 0.25 & $16^{\prime} 50^{\prime}$ \\
\hline GD2 & 2.51 & 0.7 & 3.81 & 0.049 & 0.25 & $\begin{array}{l}14^{\prime} \\
40^{\prime},\end{array}$ \\
\hline GA & -- & -- & 3.75 & 0.039 & 0.22 & $24^{\prime} 5^{\prime \prime}$ \\
\hline $\mathrm{GA}+\mathrm{GD}$ & -- & -- & 3.75 & 0.032 & 0.21 & $17^{\prime} 5^{\prime}$, \\
\hline
\end{tabular}

For Tables II-IV, in the case of using a pure GD optimization technique, the initial point is shown, and the maximum iteration number is set to 25 . For GA optimization, 25 individuals, 50 generations, and the ranges $\varepsilon^{\prime} \in[1,4], \tan \delta \in[0,2]$ for M1 and $\varepsilon^{\prime} \in[1,7]$ and $\tan \delta \in[0,5]$ for M2 are used in simulations. Finally, for the hybrid optimization strategy (GA + GD), the GA stage uses five individuals and 25 generations while GD maximum iteration number is set to 10 .

The frequency discretization number was different for the different sample geometries in measurements and simulations. For Sample 1, five frequency points were used while, for Samples 2 and 4, ten points were used. Additionally, due to the particular scattering parameters' frequency behavior of Sample 3, the frequency discretization was increased to 25 points.

From the results in Table I, it can be concluded that GA + GD is more effective than the GA or GD algorithms used individually. In fact, results demonstrate that, to obtain a similar accuracy for both dielectric constant and loss factor than the GA + GD method, the computing time is much higher for both GD or GA methods used individually. In terms of iterations, GA needs 214 generations with five individuals in each generation and pure GD uses 41 iterations versus 25 generations plus ten iterations of the GA + GD method. The number of iterations of GD is less than that for GA + GD when the initial point is close to the solution (see case GD2), but, if the initial point is far from the solution, as is the case for GD1, then this optimization algorithm does not converge. From Table I, one also can conclude that the GA algorithm is not a suitable algorithm for increasing the accuracy of the permittivity at the final stages of the optimization process. This is due to the fact that GA does not converge as a function of the objective function as fast as other methods such as GD do. Thus, using GA during the whole optimization process may lead to high computing times when a required accuracy must be reached.

From these data, several conclusions can be obtained. First of all, the convergence of the GD optimization method very strongly depends on the initial point. In fact, for all PTFE samples (Tables I-III), the algorithm does not converge to the reference value when the start point is 6.56- $j 3.92$. Nevertheless, if the start point is set to $2.51-j 0.7$, the algorithm converges in most cases. Additionally, GD convergence times also depend very much on the measurement configuration and initial point.

The GA converged to the reference value in all cases, and for all PTFE samples the dielectric constant relative error always kept below $2 \%$. Sometimes, the convergence times were even better than those provided by GD optimization as in Table I.
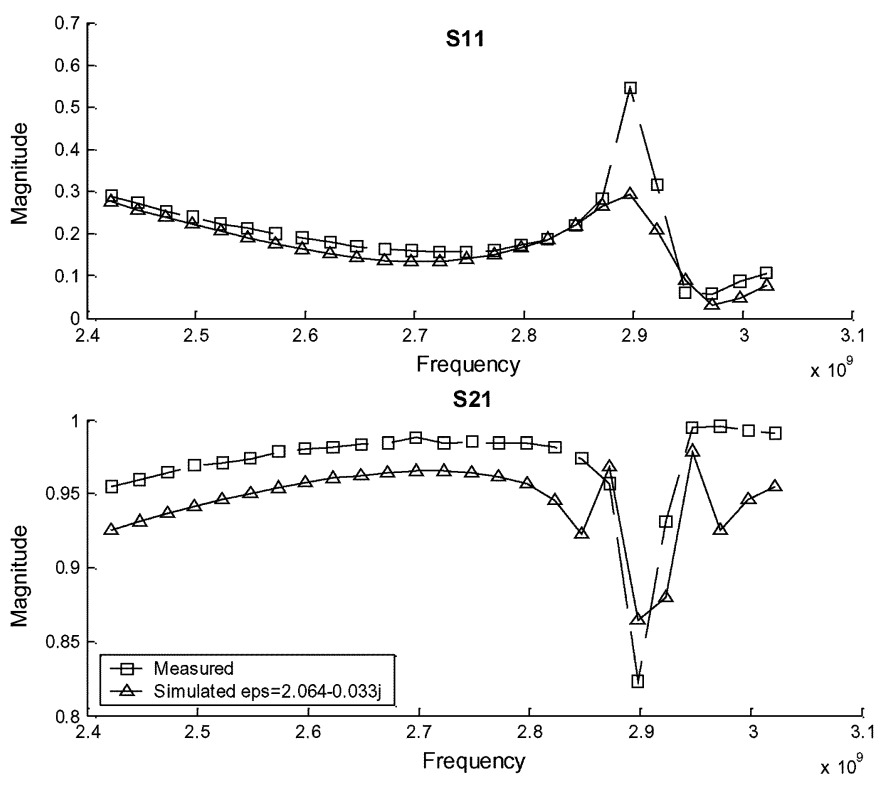

Fig. 8. Magnitude of measured and computed scattering parameters for Sample 3 and GA + GD optimum value $(\varepsilon=2.064-j 0.033)$.

Additionally, the error provided by (5) was lower with GA optimization than with GD for Tables I, III, and IV, which indicates that GA is able to match the measured and computed scattering parameters in a better way than GD technique.

The hybrid optimization strategy (GA + GD) always converged to the reference value and provided the best errors when applying (5) and, for Samples 1-3, the relative error for the dielectric constant was always lower than $2 \%$. Sometimes, the GA + GD convergence time was even better than for the GA optimization method in Tables II and IV. From these data, one can conclude that the proposed hybrid optimization technique provides the best accuracy of the optimization methods studied.

Figs. 8 and 9 compare the measured and the computed scattering parameters for the best result of the GD + GA optimization and Sample 3 both in magnitude and phase, respectively. Permittivity measurements in waveguides may introduce various complications, related both to formation of transverse modes or the appearance of resonance phenomena in measured objects. These phenomena have been observed for Sample 3 around $2.9 \mathrm{GHz}$. It can be observed that the proposed optimization strategy is able to predict the measured resonance around $2.9 \mathrm{GHz}$ and the agreement between the computed and measured scattering parameters is fine. This agreement is due to the fact that the employed software takes into account all of the possible modes that may appear in the waveguide structure through the use of Maxwell's equations.

Similar results have been obtained for all the samples. Figs. 10 and 11 illustrate the behavior of both the magnitude and phase of measured and computed parameters for Sample 4. Again, the hybrid optimization technique and the 2-D EM simulator are able to closely follow the experimental behavior of measurements.

\section{B. 3-D Multilayer Inverse Measurements}

The CST MWS commercial software has been used to carry out inverse measurements for ML1 and ML2 structures pro- 

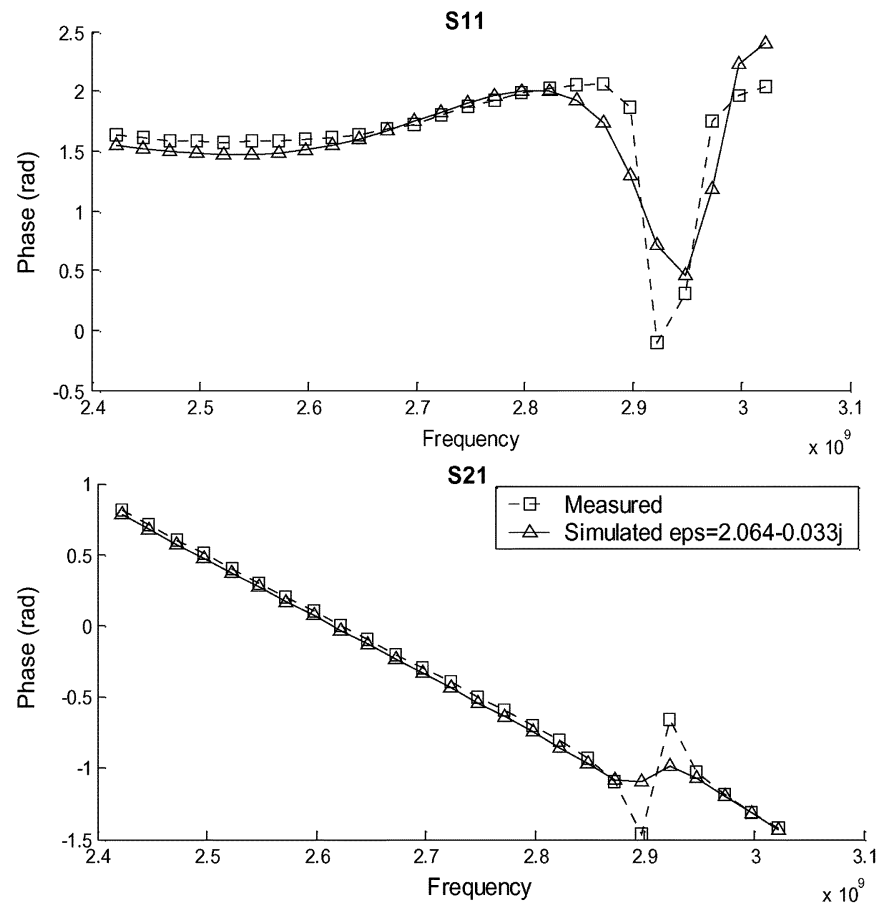

Fig. 9. Phase (radians) of measured and computed scattering parameters for Sample 3 and GA + GD optimum value $\left(\varepsilon_{r}=2.064-j 0.033\right)$.
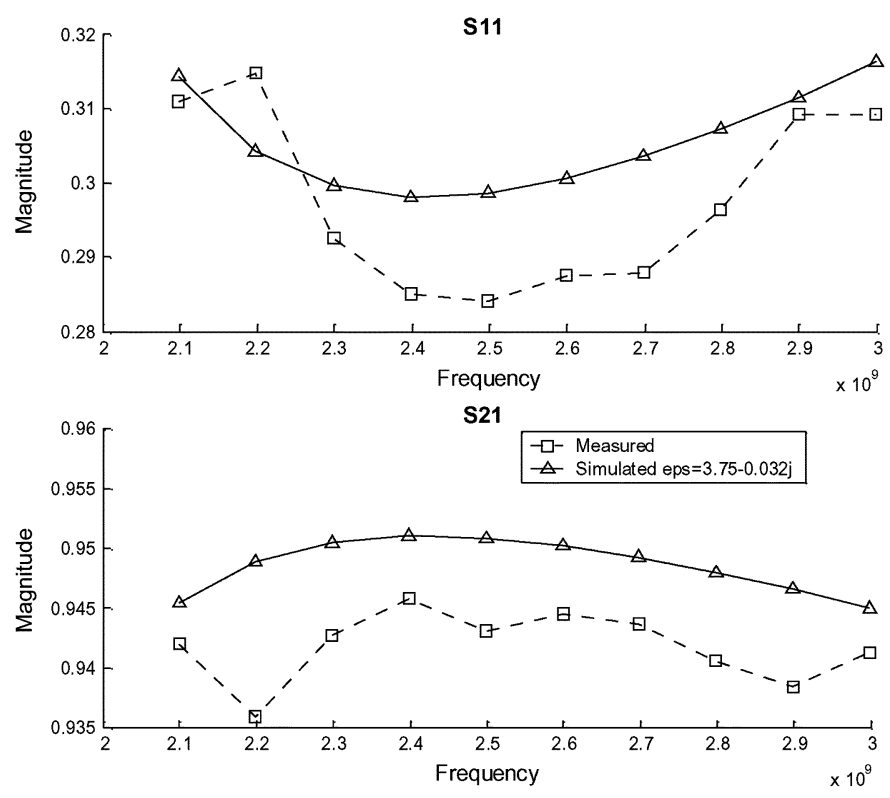

Fig. 10. Magnitude of measured and computed scattering parameters for Sample 4 and GA + GD optimum value $\left(\varepsilon_{r}=3.75-j 0.032\right)$.

viding, in this way, the individual permittivities of each element of the multilayer. The inverse measurement has combined the use of both GA and GD optimization methods, and the obtained results have been compared to Agilent 87501 commercial software based on Nicholson-Ross technique [5], which has been applied to each individual element. Both GA generation and individual numbers have been set to 50 in these measurements whereas GD maximum iteration number has been set to 100 . Table V shows the permittivity of each layer of ML1.
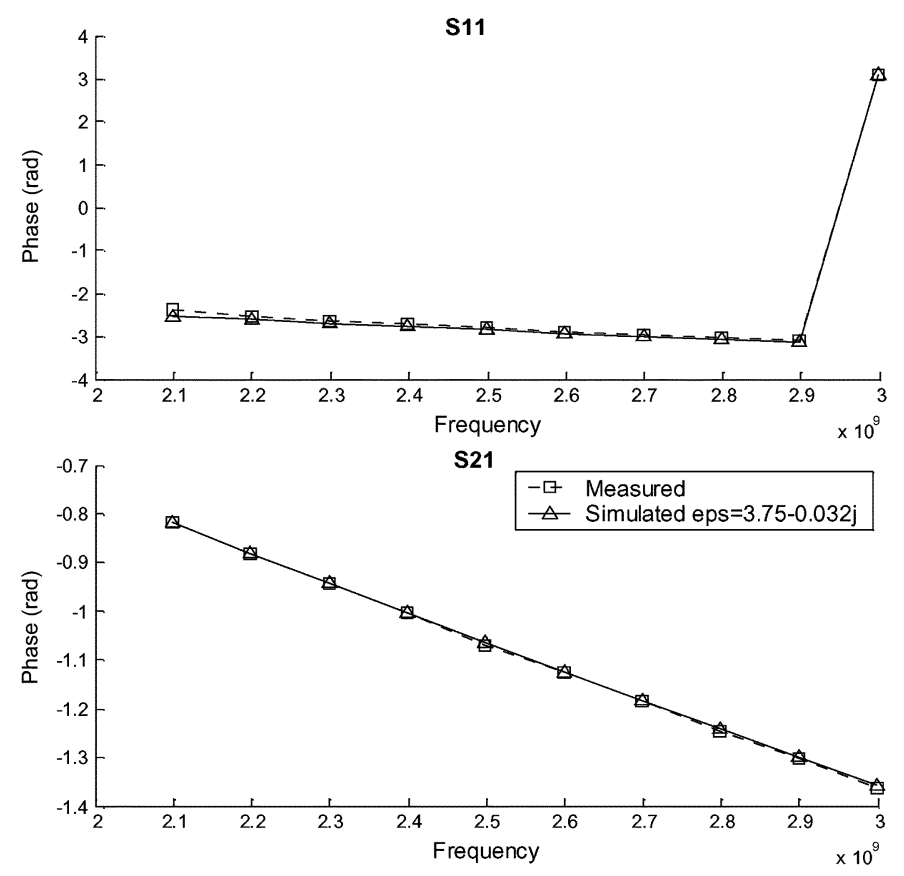

Fig. 11. Phase (radians) of measured and computed scattering parameters for Sample 4 and GA + GD optimum value $\left(\varepsilon_{r}=3.75-j 0.032\right)$.

TABLE V

PERMITTIVITY RESULTS FOR ML1

\begin{tabular}{|c|c|c|c|c|c|}
\hline Material & Method & \multicolumn{2}{|c|}{ Solution } & $\begin{array}{c}\text { Relative } \\
\text { Error (\%) }\end{array}$ & Time \\
\hline \multirow{4}{*}{$\begin{array}{l}\text { Fir Wood } \\
\text { (M3) }\end{array}$} & \multirow{2}{*}{87051} & $\varepsilon^{\prime}$ & 1.89 & - & \multirow{16}{*}{$\begin{array}{l}378^{\prime} \\
51^{\prime}\end{array}$} \\
\hline & & $\varepsilon^{\prime \prime}$ & 0.08 & - & \\
\hline & \multirow{2}{*}{$\mathrm{GA}+\mathrm{GD}$} & $\varepsilon^{\prime}$ & 1.56 & 17.46 & \\
\hline & & $\varepsilon^{\prime \prime}$ & 0.079 & - & \\
\hline \multirow{4}{*}{$\begin{array}{l}\text { Styrofoam } \\
\text { (M4) }\end{array}$} & \multirow{2}{*}{87051} & $\varepsilon^{\prime}$ & 1.59 & - & \\
\hline & & $\varepsilon^{\prime \prime}$ & 0.002 & - & \\
\hline & \multirow{2}{*}{$\mathrm{GA}+\mathrm{GD}$} & $\varepsilon^{\prime}$ & 1.7 & 6.47 & \\
\hline & & $\varepsilon^{\prime \prime}$ & 0.058 & - & \\
\hline \multirow{4}{*}{$\begin{array}{l}\text { Polyester } \\
\text { Resin (M5) }\end{array}$} & \multirow{2}{*}{87051} & $\varepsilon^{\prime}$ & 3.6 & - & \\
\hline & & $\varepsilon^{\prime \prime}$ & 0.02 & - & \\
\hline & \multirow{2}{*}{$\mathrm{GA}+\mathrm{GD}$} & $\varepsilon^{\prime}$ & 3.5 & 2.77 & \\
\hline & & $\varepsilon^{\prime \prime}$ & 0.005 & - & \\
\hline \multirow{4}{*}{ PTFE (M1) } & \multirow{2}{*}{87051} & $\varepsilon^{\prime}$ & 2.1 & - & \\
\hline & & $\varepsilon^{\prime \prime}$ & 0.01 & - & \\
\hline & \multirow{2}{*}{$\mathrm{GA}+\mathrm{GD}$} & $\varepsilon^{\prime}$ & 1.98 & 5.71 & \\
\hline & & $\varepsilon^{\prime \prime}$ & 0.021 & - & \\
\hline
\end{tabular}

From these results, it can be deduced that the method is able to provide an acceptable estimation for both the dielectric constant and loss factor of each multilayer component with a single measurement. However, the accuracy is poorer than inverse measurements carried out with single material samples.

The inverse permittivity measurement for each element of multilayer in ML2 is shown in Table VI. In this case, the relative error between the Nicholson-Ross technique, and the proposed inverse algorithm is below $8 \%$ for all materials.

Figs. 12 and 13 show the comparison of measured and computed $s_{11}$ and $s_{21}$ magnitudes and phases, respectively, for the obtained permittivity of ML1 structure. In both cases, the fitting between measured and computed scattering parameters is very good. The accuracy loss versus the previous measurements can 
TABLE VI

PERMITTIVITY RESULTS FOR ML2

\begin{tabular}{|c|c|c|c|c|c|}
\hline Material & Method & \multicolumn{2}{|c|}{ Solution } & $\begin{array}{c}\text { Relative Error } \\
(\%)\end{array}$ & Time \\
\hline \multirow{4}{*}{$\begin{array}{l}\text { Fir Wood } \\
\text { (M3) }\end{array}$} & \multirow{2}{*}{87051} & $\varepsilon^{\prime}$ & 1.89 & (2) & \multirow[t]{20}{*}{$\begin{array}{l}438^{\prime} \\
31^{\prime},\end{array}$} \\
\hline & & $\varepsilon^{\prime \prime}$ & 0.08 & - & \\
\hline & \multirow{2}{*}{$\mathrm{GA}+\mathrm{GD}$} & $\varepsilon^{\prime}$ & 1.86 & 1.58 & \\
\hline & & $\varepsilon^{\prime \prime}$ & 0.1 & - & \\
\hline \multirow{4}{*}{$\begin{array}{l}\text { Styrofoam } \\
\text { (M4) }\end{array}$} & \multirow{2}{*}{87051} & $\varepsilon^{\prime}$ & 1.59 & - & \\
\hline & & $\varepsilon "$ & 0.002 & - & \\
\hline & \multirow[t]{2}{*}{$\mathrm{GA}+\mathrm{GD}$} & $\varepsilon^{\prime}$ & 1.64 & 3.14 & \\
\hline & & $\varepsilon^{\prime \prime}$ & 0.03 & - & \\
\hline \multirow{4}{*}{$\begin{array}{l}\text { Polycarbonate } \\
\text { (M6) }\end{array}$} & \multirow{2}{*}{87051} & $\varepsilon^{\prime}$ & 2.6 & - & \\
\hline & & $\varepsilon^{\prime \prime}$ & 0.003 & - & \\
\hline & \multirow{2}{*}{$\mathrm{GA}+\mathrm{GD}$} & $\varepsilon^{\prime}$ & 2.8 & 7.69 & \\
\hline & & $\varepsilon "$ & 0.005 & - & \\
\hline \multirow{4}{*}{$\begin{array}{l}\text { Polyester } \\
\text { (M5) }\end{array}$} & \multirow{2}{*}{87051} & $\varepsilon^{\prime}$ & 3.6 & - & \\
\hline & & $\varepsilon^{\prime \prime}$ & 0.02 & - & \\
\hline & \multirow{2}{*}{$\mathrm{GA}+\mathrm{GD}$} & $\varepsilon^{\prime}$ & 3.8 & 5.55 & \\
\hline & & $\varepsilon^{\prime \prime}$ & 0.08 & - & \\
\hline \multirow{4}{*}{$\begin{array}{l}\text { PTFE } \\
\text { (M1) }\end{array}$} & \multirow{2}{*}{87051} & $\varepsilon^{\prime}$ & 2.1 & - & \\
\hline & & $\varepsilon^{\prime \prime}$ & 0.01 & - & \\
\hline & \multirow{2}{*}{$\mathrm{GA}+\mathrm{GD}$} & $\varepsilon^{\prime}$ & 1.97 & 6.19 & \\
\hline & & $\varepsilon$, & 0.02 & & \\
\hline
\end{tabular}
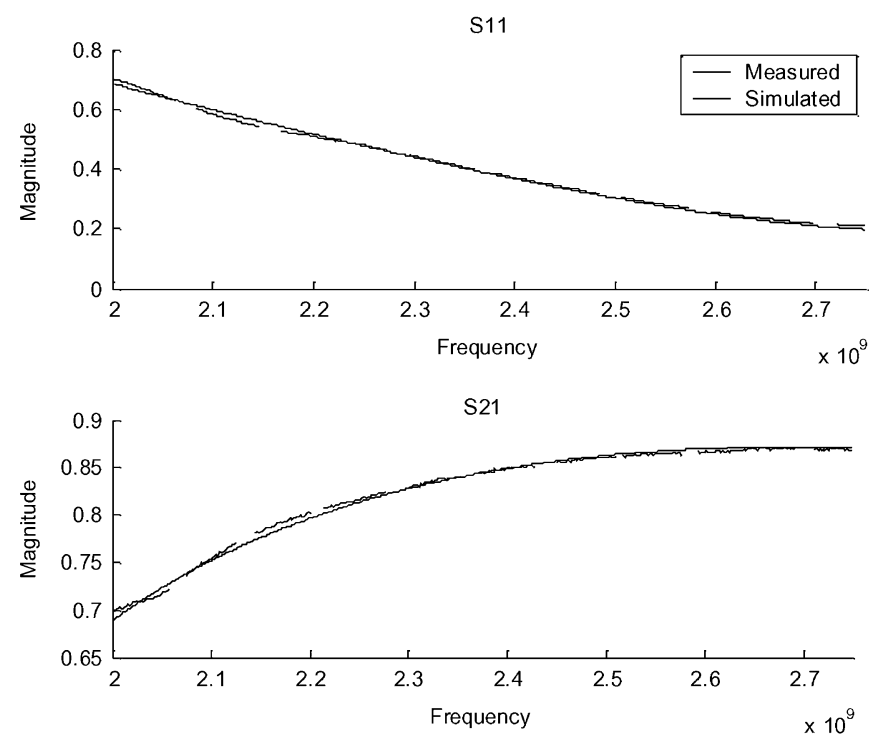

Fig. 12. Magnitude of measured and computed scattering parameters for ML1 and GA + GD permittivity optimum values, as indicated in Table V.

be explained due to the fact that the permittivity error of a single layer can be compensated by the error of another layer providing, thus, a good fitting for the scattering parameters, which are a macroscopic measurement of the whole waveguide structure. Therefore, it seems that additional measurements with different sample orientations should be carried out in order to increase the accuracy of this method when applied to dielectric multilayer structures. Additionally, the sensitivity level of the vector network analyzer also limits the accuracy of the procedure for low-loss materials, as described before.
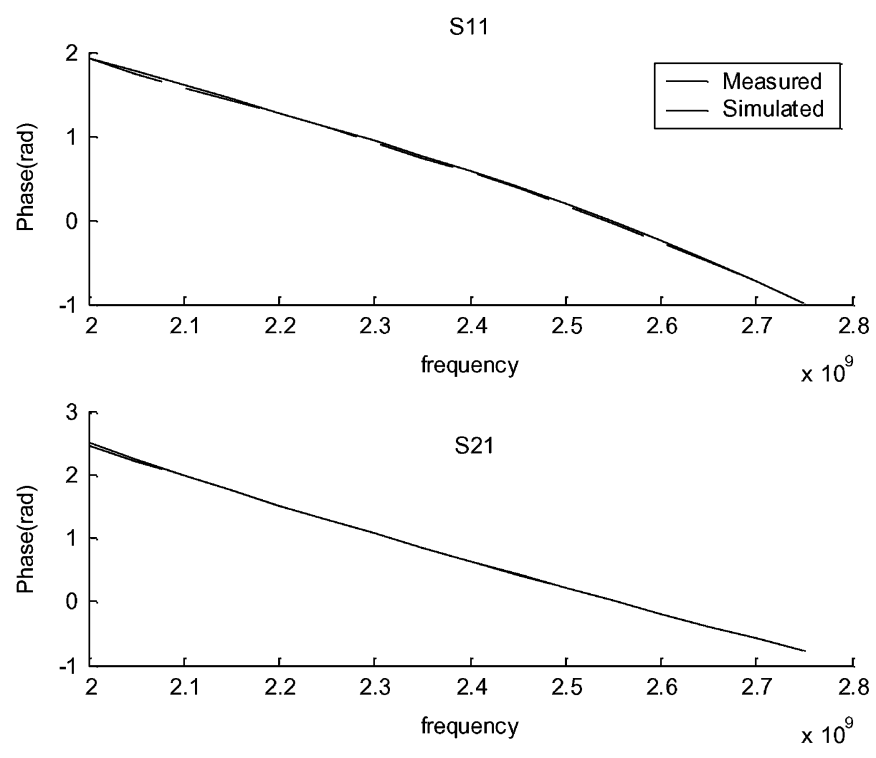

Fig. 13. Phase (radians) of measured and computed scattering parameters for ML1 and GA + GD permittivity optimum values, as indicated in Table V.

TABLE VII

PERMITTIVITY RESUlTS FOR PTFE CYLINDRICAL AND E-SHAPED SAMPLES

\begin{tabular}{|c|c|c|c|c|c|}
\hline \multirow{2}{*}{ Method } & & \multicolumn{2}{|c|}{ Solution } & \multirow{2}{*}{ Error } & \multirow{2}{*}{ Time } \\
\hline & & $\varepsilon^{\prime}$ & $\varepsilon^{\prime \prime}$ & & \\
\hline 87051 & & 2.1 & 0.01 & & \\
\hline Literature [2] & & 2.1 & 0.0003 & & \\
\hline \multirow{2}{*}{ Cylinder } & $\mathrm{GA}+\mathrm{GD}(2 \mathrm{D})$ & 2.096 & 0.0118 & 0.6826 & $13^{\prime} 10^{\prime \prime}$ \\
\hline & GA+GD (3D) & 2.079 & 0.011 & 0.25 & $754^{\prime} 47^{\prime}$ ' \\
\hline \multirow{2}{*}{ E_Shaped } & $\mathrm{GA}+\mathrm{GD}(2 \mathrm{D})$ & 2.064 & 0.033 & 7.78 & 153'20' \\
\hline & $\mathrm{GA}+\mathrm{GD}(3 \mathrm{D})$ & 2.1315 & 0.025 & 1.224 . & $500 ' 6$ ", \\
\hline
\end{tabular}

\section{3-D and 2-D Inverse Measurement Comparison}

Table VII compares the complex permittivity obtained by the 2-D simulator and the permittivity obtained in a 3-D scenario for Samples 1 and 3 and the hybrid optimization strategy $(\mathrm{GA}+\mathrm{GD})$. From these results, one can observe that the error computed by (5) is slightly smaller when using the 3-D simulator than the error provided by the 2-D simulator. However, the time needed by the $3-\mathrm{D}$ simulator increases dramatically versus the 2-D approach (in some cases, up to 50-60 times), and this does not result in appreciable dielectric constant relative error reductions.

\section{CONClusion}

A novel inverse method for permittivity estimation employing a hybrid optimization strategy based on GA and GD methods has been implemented with an in-house-made 2-D EM software and validated with measurements and 3-D EM commercial software.

Several optimization methods, namely GD, GA, and GD + GA, have been used to determine the complex permittivity by matching the measured scattering parameters with the simulated scattering parameters. The results obtained with these three methods have been compared, and, as a result, the method that provided the best accuracy was a combined strategy of the GA with the GD. The obtained results indicate 
that this combined strategy constitutes an efficient optimization tool to calculate the complex permittivity of materials.

The hybrid optimization algorithm has been applied to dielectric multilayer structures to obtain the individual permittivities of each layer. Although good matching between measured and computed scattering coefficients has been obtained, the accuracy of the elements' permittivity is worse than that obtained when applying the algorithm to homogeneous samples. Further research is envisaged in this direction to increase accuracy for these nonhomogenous structures.

The computing time comparison between 2-D and 3-D approaches indicates a time saving depending on the problem geometry, size, and frequency discretization, achieving, for some of the measurement setups, values around $98 \%$.

\section{REFERENCES}

[1] T. Jacques, Microwaves: Industrial, Scientific, and Medical Applications. Norwood, MA: Artech House, 1992.

[2] A. C. Metaxas and R. J. Meredith, Industrial Microwave Heating. London, U.K.: Peter Peregrinus, 1983.

[3] J. Baker-Jarvis, R. G. Geyer, J. H. Grosvenor Jr., M. D. Janezic, C. A. Jones, B. Riddle, C. M. Weil, and J. Kupka, "Dielectric characterization of low-loss materials. A comparison of techniques," IEEE Trans. Dielectr. Electr. Insul., vol. 5, no. 3, pp. 571-577, Aug. 1998.

[4] J. Manuel Catalá, "Estudio de estructuras guiadas monomodo para aplicaciones de caracterización dieléctrica de materiales y curado de compuestos elastoméricos a frecuencias de microondas," $\mathrm{Ph} . \mathrm{D}$. dissertation (in Spanish), Polytechnic Univ. Valencia, Valencia, Spain, Nov. 1999.

[5] A. M. Nicholson and G. F. Ross, "Measurement of the intrinsic properties of materials by the time domain techniques," IEEE Trans. Instrum. Meas., vol. MI-19, no. 6, pp. 377-382, Nov. 1970.

[6] A.-H. Boughriet, C. Legrand, and A. Chapoton, "Noniterative stable transmission/reflection method for low-loss material complex permittivity determination," IEEE Trans. Microw. Theory Tech., vol. 45, no. 1, pp. 52-57, Jan. 1997.

[7] W. B. Weir, "Automatic measurement of complex dielectric constant and permeability at microwave frequencies," Proc. IEEE, vol. 62, no. 1, pp. 33-36, Jan. 1974

[8] J. Baker, E. J. Vanzura, and W. A. Kissick, "Improved technique for determining complex permittivity with the transmission/reflection method," IEEE Trans. Microw. Theory Tech., vol. 38, no. 8, pp. 1096-1103, Aug. 1990.

[9] J. M. Catalá-Civera, A. J. Canós, F. L. Peñaranda-Foix, and E. de los Reyes Davó, "Accurate determination of the complex permittivity of materials with transmission reflection measurements in partially filled rectangular," IEEE Trans. Microw. Theory Tech., vol. 51, no. 1, pp. 17-24, Jan. 2003.

[10] D. K. Ghodgaunkar, V. V. Varadan, and V. K. Varadan, "Free space measurement of complex permittivity and complex permeability of magnetic material at microwave frequencies," IEEE Trans. Instrum. Meas., vol. 31, no. 2, pp. 387-394, Apr. 1990.

[11] P. Queffelec, P. Gelin, J. Gieraltowski, and J. Loäec, "A microstrip device for a broad band simultaneous measurement of complex permeability and permittivity," IEEE Trans. Magn., vol. 30, no. 2, pp. 224-231, Mar. 1994.

[12] P. Queffelec, M. Le-Floc'h, and P. Gelin, "A new method for determining the permeability tensor of magnetized ferrites in a wide frequency range," IEEE Trans. Microw. Theory Tech., vol. 48, no. 3, pp. 1344-1351, Aug. 2000.

[13] K. P. Thakur and W. S. Holmes, "An inverse technique to evaluate permittivity of material in a cavity," IEEE Trans. Microw. Theory Tech., vol. 49, no. 6, pp. 1129-1132, Jun. 2001.

[14] E. Eugene, E. P. Kopyt, and V. V. Yakovlev, "Determination of complex permittivity with neural networks and FDTD modeling," Microw. Opt. Technol. Lett., vol. 40, no. 3, pp. 183-188, Feb. 2004.

[15] M. Santra and K. U. Limaye, "Estimation of complex permittivity of arbitrary shape and size dielectric samples using cavity measurement technique at microwave frequencies," IEEE Trans. Microw. Theory Tech., vol. 53 , no. 2 , pp. 718-722, Feb. 2005.
[16] M. D. Deshpande, C. Jagadeswara Reddy, P. I. Tiemsin, and R. Cravey, "A new approach to estimate complex permittivity of dielectric materials at microwave frequencies using waveguide measurements," IEEE Trans. Microw. Theory Tech., vol. 45, no. 3, pp. 359-366, Mar. 1997.

[17] Y. Ramat-Samii and E. Michielssen, Electromagnetic Optimization by Genetic Algorithms. New York: Wiley.

[18] E. Domínguez-Tortajada, A. Díaz-Morcillo, J. Monzó-Cabrera, and Y. D. Sánchez-Hernández, "Application of genetic algorithms in the design of multifeeding cavities," presented at the 9th Int. Microw. RF Heating Conf., Sep. 2003.

[19] G. Fuat-Üler, O. A. Mohammed, and C.-S. Koh, "Design optimization of electrical machine using genetic algorithms," IEEE Trans. Magn., vol. 31, no. 3, pp. 2008-2011, May 1995.

[20] G. F. Üler, O. A. Moahammed, and C.-S. Koh, "Utilizing genetic algorithms for the optimal design of electromagnetics devices," IEEE Trans. Magn., vol. 30, no. 6, pp. 4296-4298, Nov. 1994.

[21] T. Zwick, J. Haala, and W. Wiesbeck, "A genetic algorithm for evaluation of material parameters of compound multilayered structures," IEEE Trans. Microw. Theory Tech., vol. 50, no. 4, pp. 1180-1187, Apr. 2002.

[22] M. Tedesco and R. Olmi, "Measuring the permittivity of thin samples in the $X$ band: A thickness-independent technique," in Proc. 3rd Int Microw. Millimeter Wave Technol. Conf., Beijing, China, Aug. 2002, pp. 118-121. 2002.

[23] T. Chiu, "Dielectric constant measurement technique for a dielectric strip using a rectangular waveguide," IEEE Trans. Instrum. Meas., vol. 52, no. 5, pp. 1501-1508, Oct. 2003.

[24] K. Huang, X. Cao, C. Liu, and X.-B. Xu, "Measurement/computation of effective permittivity of dilute solution in saponification reaction," IEEE Trans. Microw. Theory Tech., vol. 51, no. 10, pp. 2106-2110, Oct. 2003.

[25] R. Omi, M. Bini, A. Ignesti, and C. Riminesi, "Non destructive measurement of solid materials," Meas. Sci. Technol., vol. 11, pp. 1623-1629, 2000.

[26] M. E. Requena-Pérez, A. Díaz-Morcillo, and J. Monzó-Cabrera, "Accurate permittivity measurement by means of inverse calculation based on genetic algorithms," in Proc. IEEE AP-S Int. USNC/URSI Nat. Radio Sci. Meeting Symp., Monterey, CA, Jun. 2004, pp. 511-514.

[27] Partial Differential Equation Toolbox User's Guide, The MathWorks, Natick, MA, 2004. [Online]. Available: www.mathworks.com.

[28] HF Design and Analysis Manual, Version 4, CST Microwave Studio, Darmstadt, Germany, 2002.

[29] C. R. Houck, J. A. Joines, and M. G. Kay, "A genetic algorithm for function optimization: A MATLAB implementation," The MathWorks, Natick, MA, NCSU-IE TR 95-09, 1995.

[30] Optimization Toolbox User's Guide, The MathWorks, Natick, MA, 2004. [Online]. Available: www.mathworks.com.

[31] J. Heistermann, A Mixed Genetic Approach to the Optimization of Neural Controllers. New York: IEEE Press, 1992.

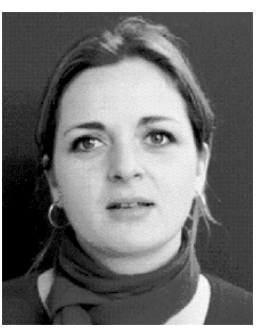

María E. Requena-Pérez was born in Alicante, Spain. She received the Dipl. Ing. degree in telecommunications engineering from the Universidad Politécnica de Valencia, Valencia, Spain, and is currently working toward the Ph.D. degree at the Universidad Politécnica de Cartagena, Cartagena, Spain.

In 2000, she joined the Department of Information Technologies and Communications, Universidad Politécnica de Cartagena. Her current research areas are microwave-assisted heating and drying processes, microwave applicator design, permittivity measurement and optimization, and numerical techniques in electromagnetics.

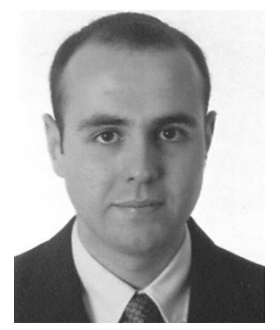

Antonio Albero-Ortiz was born in Murcia, Spain, on July 1981. He received the Dipl. Ing. degrees in telecommunications engineering from the Universidad Politécnica de Cartagena, Cartagena, Spain, in 2005.

In 2004, he joined the Department of Information Technologies and Communications, Universidad Politécnica de Cartagena. 


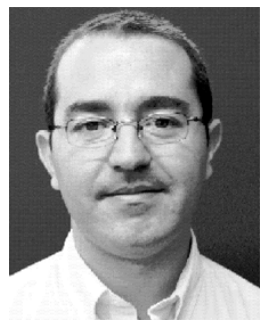

Juan Monzó-Cabrera was born in Elda (Alicante), Spain, in January 1973. He received the Dipl. Ing. and Ph.D. degrees in telecommunications engineering from the Universidad Politécnica de Valencia, Valencia, Spain, in 1998 and 2002, respectively.

From 1999 to 2000, he was a Research Assistant with the Microwave Heating Group (GCM). In 2000, he joined the Departamento de Teoría de la Señal y Radiocomunicaciones, Universidad Politécnica de Cartagena, Cartagena, Spain, as an Associate Lecturer, and is currently an Associate Lecturer with the Departamento de Tecnologías de la Información y Comunicaciones. He has coauthored over 30 papers in refereed journals and conference proceedings. He holds one patent regarding microwave heating industrial processes. His current research areas cover microwave-assisted heating and drying processes, microwave applicator design and optimization and numerical techniques in electromagnetics.

Dr. Monzó-Cabrera is a member of the Association of Microwave Power in Europe for Research and Education (AMPERE), a European-based organization devoted to the promotion of RF and microwave energy. He is a reviewer of several international journals.

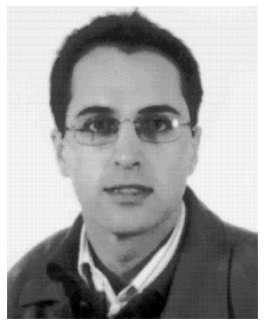

Alejandro Díaz-Morcillo (S'95-M'02) was born in Albacete, Spain, in 1971. He received the Ingeniero (M.Eng.) and Doctor Ingeniero (Ph.D.) degrees in telecommunication engineering from the Polytechnic University of Valencia (UPV), Valencia, Spain, in 1995 and 2000, respectively.

From 1996 to 1999, he was a Research Assistant with the Department of Communications, UPV. In 1999, he joined the Department of Information Technologies and Communications, Universidad Politécnica de Cartagena (UPCT), Cartagena, Spain, as a Teaching Assistant, where, since 2001, he has been an Associate Professor. He leads the Electromagnetics and Matter Research Group, UPCT. His main research interest focuses on numerical methods in electromagnetics and industrial microwave heating systems. 